\title{
Exploring new pathways in the treatment of hepatocellular cancer
}

\author{
Eukasz Masior ${ }^{1}$, Michał Grąt ${ }^{2}$ \\ ${ }^{1}$ Department of General, Vascular and Oncological Surgery, Medical University of Warsaw, Warsaw, Poland; ${ }^{2}$ Department of General, Transplant \\ and Liver Surgery, Medical University of Warsaw, Warsaw, Poland \\ Correspondence to: Łukasz Masior. Department of General, Vascular and Oncological Surgery, Medical University of Warsaw, 00-739, Stępińska Street \\ 19/25, Warsaw, Poland. Email: lmasior@gmail.com. \\ Provenance and Peer Review: This article was commissioned by the editorial office, Annals of Translational Medicine. The article did not undergo \\ external peer review. \\ Comment on: Feng LH, Sun HC, Zhu XD, et al. Renin-angiotensin inhibitors were associated with improving outcomes of hepatocellular carcinoma \\ with primary hypertension after hepatectomy. Ann Transl Med 2019;7:739.
}

Submitted Apr 29, 2020. Accepted for publication May 09, 2020.

doi: $10.21037 /$ atm-20-3629

View this article at: http://dx.doi.org/10.21037/atm-20-3629

The pandemic caused by coronavirus responsible for COVID-19 disease is obviously the focus of interest worldwide. Round-the-clock thousands of people work to ensure that the number of new cases and deaths is kept low, and that effective drugs and vaccines will be available in the foreseeable future. However, pandemic has not replaced other global medical challenges, such as hepatocellular cancer (HCC) (1). Effective treatment of this disease is still based mainly on extensive surgical procedures-liver resection and transplantation, which are the only potentially curative treatment options.

Liver resection provides comparable life-expectancy to patients with chronic hepatitis/cirrhosis (without cancer) in only $26.3 \%$ of patients with HCC. These chances obviously increase over time. Thus, patients who survived 2 years without recurrence have $50 \%$ probability of statistical cure, and beyond 8.9 years it reaches up to $95 \%$ (2). Opinions on the greater efficacy of anatomical resections have recently been called into question even in patients with microportal invasion. This was demonstrated by analyzing a group of 546 patients undergoing liver resection in Japan. Overall and recurrence-free survival did not differ significantly between patients who underwent anatomical and nonanatomical resection (3). On the other hand, aggressive surgical strategies provide satisfactory results, also in groups of patients who have traditionally been considered as having a poor prognosis. This group includes patients with bile duct tumor thrombus. It seems that biliary tract involvement alone has less prognostic significance than classic risk factors, including microvascular invasion (MVI). Therefore, extensive hepatectomies combined with bile duct resection allow to achieve a 5 -year survival of approximately $40 \%$ in a selected group of patients (4). MVI is still considered by most authors to be one of the strongest, negative risk factors. Until now, the invasion was assessed based on histopathological examination of surgical specimen. Nowadays, MVI can be predicted with sensitivity and specificity at around $80 \%$ by the analysis of circulating tumor DNA extracted from the peripheral blood samples (5). This knowledge can obviously affect treatment strategy towards more tailored approach. An example of such an individualized strategy is the use of adjuvant TACE (Trans-arterial chemoembolisation). A meta-analysis from China showed a significant improvement in overall and recurrence-free survival only in the group of patients with tumors greater than $5 \mathrm{~cm}$, multifocal lesions and the presence of MVI. Furthermore, the use of TACE in patients without MVI worsened recurrence-free survival (HR 1.20; 95\% CI: 1.03-1.39; $\mathrm{P}=0.002$ ). This may be associated with hypoxia, increases in the concentrations of hypoxia-induced factor 1a (HIF-1a) and vascular endothelial growth factor (VEGF) and stimulation of neoangiogenesis (6).

The natural limitation of liver resection related mostly to the presence of liver cirrhosis in a significant proportion of patients, paved the way for liver transplantation as an optimal treatment strategy. Qualification process is still based mainly on Milan criteria (1 tumor no larger than $5 \mathrm{~cm}$ or 3 tumors with a maximum diameter of $3 \mathrm{~cm}$ each) providing 
excellent results with statistical cure possible in over $70 \%$ of patients $(7,8)$. However, more often the cornerstone at the time of qualification is the assessment of tumor biology, mainly based on pre-transplant AFP concentration. Another important element is the response to neoadjuvant therapy, which is crucial especially for patients who are primarily outside the Milan criteria. Classic morphological parameters such as diameter and number of lesions seem to be of lesser importance nowadays (7). This applies even more to patients who are transplanted from living donors, where the criteria are more liberal (7). Turning more towards biology instead of tumor morphology allows for excellent results even in patients with macrovascular involvement, a parameter which until now was considered as a classic contraindication to transplantation. In case of the disappearance of vascular invasion in pre-transplant imaging studies (after neoadjuvant therapy) with concomitant AFP concentration $<10 \mathrm{ng} / \mathrm{mL}$, the 5 -year survival is over $80 \%$ (9). Possibility of achieving very good results with so-called salvage transplantation highlights the complementary role of liver resection and transplantation $(7,10)$. Importantly, the primary liver resection with a minimally invasive technique may play an important role in this clinical scenario, allowing for impressive 80\% 5 -year long-term survival. Better results of salvage transplantation in patients who have undergone previously laparoscopic resection may be associated with less difficulty during the first stage of transplant procedure (explantation), which translates into shorter surgery times, shorter cold ischemia times, fewer transfusions and less postoperative complications (10). Of course, salvage transplantation is an option only for a fraction of patients. In the case of limited liver-only recurrence, patients with preserved liver function can be successfully treated with re-resection or thermoablation with 5-year survival expected in approximately $40 \%$ of patients. In general these techniques provide similar results, however the superiority of resection was shown in a subgroup of patients with tumors larger than $3 \mathrm{~cm}$ and AFP concentration higher than $200 \mathrm{ng} / \mathrm{mL}$ (11).

Systemic treatment with Sorafenib is the standard option for patients not eligible for invasive therapy. Knowledge about immunotherapy, which has been developing more dynamically in recent years, also plays its role in patients with HCC (12). Pembrolizumab, which is an anti-programmed death-1 (PD-1) monoclonal antibody, was evaluated in a phase III randomized KEYNOTE-240 trial. The study included patients not amenable to curative approach, previously treated with Sorafenib, preserved liver function and tumors in stage $\mathrm{B}$ or $\mathrm{C}$ according to Barcelona Clinic Liver Cancer (BCLC) classification. Although the pre- defined statistical significance levels were not achieved, the drug has proven its efficacy (median survival 13.9 months) and was characterized by low toxicity as well (13).

The still unsatisfactory cancer-related outcomes and the unparalleled curiosity of oncologists have allowed the exploration of territories that until now were the domain of other specialists. The renin-angiotensin system (RAS) has been deeply explored in many cardiology studies so far. Drugs blocking various elements of this system are commonly used in the treatment of hypertension and other cardiovascular diseases. However, it turns out that this pathway also plays an important role in the process of cancerogenesis and its inhibition may improve the results of treatment of certain cancers (14). These drugs have already been successfully used in prospective studies, e.g., in neoadjuvant setting in patients with pancreatic cancer. Despite good safety profile, impact of losartan on final outcomes could not be evaluated based on the single-arm study design (15). Meta-analysis from 2019 revealed that this type of hypotensive therapy significantly improves survival in patients with digestive tract malignancies (16). Similar effect was observed in patients with kidney cancer based on recent data (17). Prostate cancer is another potentially targeted tumor (18).

Data regarding influence of RAS inhibitors on outcomes of patients with HCC are lacking and are mostly based on retrospective studies. In paper of Pinter et al., influence of RAS inhibitors were analyzed in two group of patients at the Medical University of Vienna (Vienna group) and, to validate the results, at the Johannes Gutenberg University in Mainz (Mainz group). In Vienna group patients received Sorafenib, experimental therapy or best supportive care, whereas in Mainz only Sorafenib was applied. Administration of RAS inhibitors was associated with significantly longer overall survival in both the Vienna group (11.9 vs. 6.8 months, $\mathrm{P}=0.014)$ and the Mainz group (13.2 vs. 8 months, $\mathrm{P}=0.005)$. Moreover, in both cohorts RAS inhibitors were found to be significant factors linked to better overall survival in multivariable analyses (19). In another study, different hypotensive drugs were assessed in 153 patients with BCLC 0/A HCC treated by radiofrequency ablation (RFA). Median overall survival in patients using sartans was significantly longer ( 84 months) than in patients treated by ACE (angiotensin-converting enzyme) inhibitors (72 months) and those using other medications (48 months; $\mathrm{P}=0.02$ ). In multivariable analysis, sartans administration was an independent predictor of better overall survival (HR 0.39, CI: 95\% 0.22-0.66, P=0.002) (20). Small randomized paper 
from the Nara Medical University evaluated 87 patients with HCC treated with radical RFA according to local guidelines (21). As an adjuvant strategy ACE inhibitors and vitamin $\mathrm{K}$ were given in the treatment arm, while control group received no treatment, including placebo. Combined treatment was associated with lower recurrence rates when compared to the control group (36\% vs. $72 \%$ after 2 years; $\mathrm{P}<0.01)$. Another study conducted by the same group from Japan evaluated 110 patients with HCC and insulin resistance (22). Similarly, all patients were radically treated by percutaneous RFA. Again lower cumulative recurrence rates were observed among patients who simultaneously used angiotensin-converting enzyme inhibitor (ACE-I) and branched-chain amino acid (BCAA). Significant decrease in serum VEGF levels were observed in both studies, pointing out to an important role of angiogenesis attenuation in reducing the risk of HCC relapse. Potential role of RAS inhibition could be further extent into chemoprevention of this cancer. However, caution is advocated in HCV patients without cirrhosis, diabetes and hyperlipidemia, in whom exposure to RAS inhibitors may even increase the risk of HCC development $(23,24)$.

In the manuscript of Feng et al. published in one of the recent issues of Annals of Translational Medicine, 387 patients with primary hypertension who underwent radical liver resection due to HCC were analyzed (25). The study included patients with BCLC stage 0, A and B and with liver function assessed as Child-Pugh A or B. 144 patients (37.2\%) used RAS inhibitors (RASI group), the rest of the cohort used other hypotensive medications (B-blockers, CCB-calcium channel blockers; non-RASI group). Basic characteristics did not differ significantly between groups, however data specifically referring to cardiovascular and renal comorbidities were not presented. All clinically significant oncological and liver-related variables were included in statistical analyses. RASI administration was associated with recurrence rates $10.4 \%, 27.4 \%$ and $38.2 \%$ after the first, third and fifth year, respectively. Accordingly, in the non-RASI group recurrences were diagnosed in $19.0 \%, 42.5 \%$ and $56.5 \%$ patients after the first, third and fifth year, respectively $(\mathrm{P}<0.001)$. Similarly, overall survival after the first, third and fifth year was better in patients treated with RASI $(96.5 \%, 90.2 \%$ and $82.3 \%$, respectively) than in cohort using non-RASI $(95.1 \%, 79.8 \%$ and $64.2 \%$, respectively; $\mathrm{P}<0.001)$. In multivariate analysis, the use of RASI was found as having protective effect on both recurrence-free (HR 0.52, 95\% CI: $0.38-0.70, \mathrm{P}<0.001)$ and overall survival (HR 0.50, 95\% CI: 0.34-0.74, $\mathrm{P}<0.001$ ). Moreover, lower incidence of extrahepatic metastases were observed among patients managed by RASI $(2.8 \% v s$. $7.8 \%, \mathrm{P}<0.043)$. No significant differences in recurrence rates and overall survival were seen when outcomes were compared based on whether or not patients used different types of non-RASI (B-blocker vs. non-B-blocker; CCB $v s$. non-CCB), as well as in patients using different types of RASI. The mechanism of potential beneficial effects of RAS inhibitors is multifactorial and still unexplored. It may include, among other, inhibition of angiogenesis, modification of the immune response or inhibition of tumor stem cell maturation (14). In the context of HCC, the role of RASI may also be associated with inhibition of progression of liver fibrosis and subsequent improvement of organ function, which is also emphasized by Feng et al. (25).

Despite these encouraging results the published article has certain limitations. Its retrospective nature is prone to obvious biases, thus key elements related to treatment duration, drugs dosages etc. could not be properly assessed. Number of patients in the RASI group was relatively small and potential interactions between different medications used in both groups could still play an important role. Further, in order to provide a better insight into the real oncological effects of RAS inhibitors, exact data on the reasons for selection of particular therapeutic regimen for hypertension are necessary. Otherwise, the selection bias associated with other conditions, such as comorbidities, compliance, healthcare access, severity of portal hypertension, and severity of fibrosis, blurs the picture of the association between the RAS inhibitors and outcomes HCC patients undergoing surgical therapy.

Undoubtedly, we are at the beginning of the road to full exploration of RAS inhibitors as a cancer-specific drugs. However, the range of potential opportunities that this pathway could reveal in the future is really exciting. Therefore, the authors should be congratulated for having enriched our knowledge on this subject in a clear and methodologically appropriate manner. Nevertheless, observations on the potential benefits of RAS inhibitors in HCC patients undergoing liver resection need to be confirmed in prospective randomized clinical trials.

\section{Acknowledgments}

MG received a stipend for outstanding young scientists from the Ministry of Science and Higher Education of the 
Republic of Poland (571/STYP/14/2019).

Funding: None.

\section{Footnote}

Conflicts of Interest: Both authors have completed the ICMJE uniform disclosure form (available at http://dx.doi. org/10.21037/atm-20-3629). MG serves as an unpaid editorial board member of Annals of Translational Medicine from Feb 2019 to Jan 2021. MG reports other from Ministry of Science and Higher Education of the Republic of Poland, during the conduct of the study. The other author has no conflicts of interest to declare.

Ethical Statement: The authors are accountable for all aspects of the work in ensuring that questions related to the accuracy or integrity of any part of the work are appropriately investigated and resolved.

Open Access Statement: This is an Open Access article distributed in accordance with the Creative Commons Attribution-NonCommercial-NoDerivs 4.0 International License (CC BY-NC-ND 4.0), which permits the noncommercial replication and distribution of the article with the strict proviso that no changes or edits are made and the original work is properly cited (including links to both the formal publication through the relevant DOI and the license). See: https://creativecommons.org/licenses/by-nc-nd/4.0/.

\section{References}

1. Global Burden of Disease Cancer Collaboration, Fitzmaurice C, Akinyemiju TF, et al. Global, Regional, and National Cancer Incidence, Mortality, Years of Life Lost, Years Lived With Disability, and Disability-Adjusted LifeYears for 29 Cancer Groups, 1990 to 2016: A Systematic Analysis for the Global Burden of Disease Study. JAMA Oncol 2018;4:1553-68.

2. Cucchetti A, Zhong J, Berhane S, et al. The chances of hepatic resection curing hepatocellular carcinoma. J Hepatol 2020;72:711-7.

3. Hidaka M, Eguchi S, Okuda K, et al. Impact of Anatomical Resection for Hepatocellular Carcinoma With Microportal Invasion (vp1): A Multi-institutional Study by the Kyushu Study Group of Liver Surgery. Ann Surg 2020;271:339-46.

4. Kim DS, Kim BW, Hatano E, et al. Surgical Outcomes of Hepatocellular Carcinoma With Bile Duct Tumor Thrombus: A Korea-Japan Multicenter Study. Ann Surg
2020;271:913-21.

5. Wang D, Xu Y, Goldstein JB, et al. Preoperative evaluation of microvascular invasion with circulating tumor DNA in operable hepatocellular carcinoma. Liver Int 2020;8:237.

6. Chen W, Ma T, Zhang J, et al. A systematic review and meta-analysis of adjuvant transarterial chemoembolization after curative resection for patients with hepatocellular carcinoma. HPB (Oxford) 2020;22:795-808.

7. Mehta N, Bhangui P, Yao FY, et al. Liver Transplantation for Hepatocellular Carcinoma. Working Group Report from the ILTS Transplant Oncology Consensus Conference. Transplantation 2020;104:1136-42.

8. Pinna AD, Yang T, Mazzaferro V, et al. Liver Transplantation and Hepatic Resection can Achieve Cure for Hepatocellular Carcinoma. Ann Surg 2018;268:868-75.

9. Assalino $M$, Terraz $S$, Grat $M$, et al. Liver transplantation for hepatocellular carcinoma after successful treatment of macrovascular invasion - a multi-center retrospective cohort study. Transpl Int 2020;33:567-75.

10. Levi Sandri GB, Lai Q, Ravaioli M, et al. The role of salvage transplantation in patients initially treated with open vs minimally invasive liver surgery: an intention-totreat analysis. Liver Transpl 2020;26:878-87.

11. Xia Y, Li J, Liu G, et al. Long-term Effects of Repeat Hepatectomy vs Percutaneous Radiofrequency Ablation Among Patients With Recurrent Hepatocellular Carcinoma: A Randomized Clinical Trial. JAMA Oncol 2019;6:255-63.

12. Nakano S, Eso Y, Okada H, et al. Recent Advances in Immunotherapy for Hepatocellular Carcinoma. Cancers (Basel) 2020;12:E775.

13. Finn RS, Ryoo BY, Merle P, et al. Pembrolizumab As Second-Line Therapy in Patients With Advanced Hepatocellular Carcinoma in KEYNOTE-240: A Randomized, Double-Blind, Phase III Trial. J Clin Oncol 2020;38:193-202.

14. Perini MV, Dmello RS, Nero TL, et al. Evaluating the benefits of renin-angiotensin system inhibitors as cancer treatments. Pharmacol Ther 2020. [Epub ahead of print].

15. Murphy JE, Wo JY, Ryan DP, et al. Total Neoadjuvant Therapy With FOLFIRINOX in Combination With Losartan Followed by Chemoradiotherapy for Locally Advanced Pancreatic Cancer: A Phase 2 Clinical Trial. JAMA Oncol 2019;5:1020-7.

16. Zhou Q, Chen DS, Xin L, et al. The renin-angiotensin system blockers and survival in digestive system malignancies: A systematic review and meta-analysis. 
Medicine (Baltimore) 2020;99:e19075.

17. Asgharzadeh F, Hashemzehi M, Moradi-Marjaneh R, et al. Angiotensin-converting enzyme inhibitors and angiotensin receptor blockers as therapeutic options in the treatment of renal cancer: A meta-analysis. Life Sci 2020;242:117181.

18. Santala EE, Rannikko A, Murtola TJ. Antihypertensive drugs and prostate cancer survival after radical prostatectomy in Finland-A nationwide cohort study. Int J Cancer 2019;144:440-7.

19. Pinter M, Weinmann A, Wörns MA, et al. Use of inhibitors of the renin-angiotensin system is associated with longer survival in patients with hepatocellular carcinoma. United European Gastroenterol J 2017;5:987-96.

20. Facciorusso A, Del Prete V, Crucinio N, et al. Angiotensin receptor blockers improve survival outcomes after radiofrequency ablation in hepatocarcinoma patients. J Gastroenterol Hepatol 2015;30:1643-50.

21. Yoshiji H, Noguchi R, Toyohara M, et al. Combination of vitamin $\mathrm{K} 2$ and angiotensin-converting enzyme inhibitor ameliorates cumulative recurrence of hepatocellular

Cite this article as: Masior $€$, Grąt M. Exploring new pathways in the treatment of hepatocellular cancer. Ann Transl Med 2020;8(16):980. doi: 10.21037/atm-20-3629 carcinoma. J Hepatol 2009;51:315-21.

22. Yoshiji H, Noguchi R, Ikenaka Y, et al. Combination of branched-chain amino acids and angiotensin-converting enzyme inhibitor suppresses the cumulative recurrence of hepatocellular carcinoma: a randomized control trial. Oncol Rep 2011;26:1547-53.

23. Barone M, Viggiani MT, Losurdo G, et al. Systematic review: Renin-angiotensin system inhibitors in chemoprevention of hepatocellular carcinoma. World J Gastroenterol.2019;25:2524-38.

24. Ho CM, Lee CH, Lee MC, et al. Comparative effectiveness of angiotensin-converting enzyme inhibitors and angiotensin II receptor blockers in chemoprevention of hepatocellular carcinoma: a nationwide high-risk cohort study. BMC Cancer 2018;18:401.

25. Feng LH, Sun HC, Zhu XD, et al. Renin-angiotensin inhibitors were associated with improving outcomes of hepatocellular carcinoma with primary hypertension after hepatectomy. Ann Transl Med 2019;7:739. 